\title{
ON CERTAIN ELEMENTARY EXTENSIONS OF MODELS OF SET THEORY
}

BY

\author{
ALI ENAYAT
}

\begin{abstract}
In $\$ 1$ we study two canonical methods of producing models of ZFC with no elementary end extensions. $§ 2$ is devoted to certain "completeness" theorems dealing with elementary extensions, e.g., using $\diamond_{\omega_{1}}$ we show that for a consistent $T \supseteq$ ZFC the property "Every model $\mathfrak{A}$ of $T$ has an elementary extension fixing $\omega^{\mathfrak{A}}$ " is equivalent to $T \vdash$ "There exists an uncountable measurable cardinal". We also give characterizations of $T \vdash$ " $\kappa$ is weakly compact" and $T \vdash$ " $\kappa$ is measurable" in terms of elementary extensions.
\end{abstract}

Introduction. This paper deals with the study of elementary extensions of models of set theory, an area first systematically investigated by Keisler and Morley in $[\mathbf{K M}]$, and later by Keisler and Silver in [KS]. Two recent contributions come from J. Hutchinson [H1] and M. Kaufmann [Ka]. Apart from its intrinsic interest as a chapter of (western) model theory, the study of elementary extensions of models of set theory has also benefitted general model theory, often by yielding soft proofs of known theorems, and sometimes, providing new results. The interested reader is referred to Hutchinson [H2] for a sample of such applications.

In $\S 1$ we discuss models of set theory with no elementary end extensions. Specifically, we provide two distinct methods which allow one to construct models of any consistent theory $T \supseteq \mathrm{ZFC}$ with no elementary end extensions. This section is closely tied with the work of Kaufmann in [Ka].

$\$ 2$ deals with certain "completeness" theorems, and once more reminds us of the nice behavior of first-order logic. More precisely, we identify, in our "completeness" theorems, first-order equivalents of certain second-order properties of set theories. For example, in Theorem 2.17, we show that for a consistent theory $T \supseteq$ ZFC the second-order property "Every model $\mathfrak{A}$ of $T$ has an elementary extension fixing $\omega^{\mathfrak{A}}$ " is equivalent to

$$
T \vdash \text { “There exists an uncountable measurable cardinal". }
$$

In contrast, in Theorem 2.6 we present an "incompleteness" phenomenon found by Kunen.

The somewhat spartan models first introduced by Keisler and Kunen in [K3], and later generalized by Shelah in [S] and Rubin in [RS], play a key role in §2. Because

Received by the editors July 25,1983 . The main results of this paper were presented to the annual meeting of the Association for Symbolic Logic on December 29, 1983 in Boston.

1980 Mathematics Subject Classification. Primary 02H05; Secondary 02K35.

(c) 1984 American Mathematical Society $0002-9947 / 84 \$ 1.00+\$ .25$ per page 
of their dependence on a result of Rubin and Shelah [RS], certain results in this section require $\diamond_{\omega_{1}}$. We suspect that these results are true outright in ZFC. Some of the results of this paper were announced in $[\mathbf{E}]$.

0. Notation and conventions. (a) Language. Our language consists of $\{\in\}$ and possibly countably many constant symbols. All our results remain true if finitely many relation or function symbols are present, provided of course, they appear in the replacement scheme.

(b) Structures. Models of set theory are of the form $\mathfrak{A}=\langle A, E, \ldots\rangle$ where $E$ is a binary (usually non-well-founded) relation on $A$. Universes of structures are usually denoted by the corresponding Roman letters. Given a member $c$ of $\mathfrak{A}$ we denote the extension of $c$ in $\mathfrak{A}$ by $c_{E}$, hence $c_{E}=\{b \in A: b E c\}$. If $\alpha$ is an ordinal of $\mathfrak{A}, \mathfrak{U}_{\alpha}$ refers to what $\mathfrak{A}$ 'thinks' is $\langle R(\alpha), \in\rangle$. Any order-theoretic attribute of a model $\mathfrak{A}$ must be understood as the attribute of its linearly ordered set of ordinals $\langle\operatorname{Ord}(\mathfrak{A}), E\rangle$, hence $\operatorname{cf}(\mathfrak{H})>\omega$ means $\operatorname{cf}(\langle\operatorname{Ord}(\mathfrak{H}), E\rangle)>\omega$. Given models $\mathfrak{A}$ $=\langle A, E, \ldots\rangle \subseteq \mathfrak{B}=\langle B, F, \ldots\rangle$ we say that $\mathfrak{P}$ fixes an element $c$ of $\mathfrak{U}$ if $c_{E}=c_{F}$, else $\mathfrak{B}$ is said to enlarge $c$. If $\mathfrak{B}$ fixes all elements of $\mathscr{A}$ then we say that $\mathfrak{X}$ is an end extension of $\mathfrak{A}$, denoted $\mathfrak{A} \subset \mathfrak{P}$. $\mathfrak{B}$ is said to rank extend $\mathfrak{A}$, denoted $\mathfrak{A} \subset \mathfrak{F}$, if $\mathfrak{U} \subset \mathbb{W}$ and every new element of $\mathcal{H}$ is above $\mathfrak{H}$, i.e., if $b \in B \backslash A$ then $\operatorname{rank}(b) \in$ $B \backslash A$. It is important to note that $\mathfrak{H} \underset{e}{\prec} \mathfrak{W}$ implies $\mathfrak{H} r \mathfrak{r}$. Lastly $\mathfrak{A} \prec \mathfrak{W}$ means that $\mathfrak{A}$ is a $\Sigma_{n}$-elementary submodel of $\mathfrak{B}$. We usually abbreviate the clause "elementary end extension" by "e.e.e."

1. Models with no rank extensions. Following the classical theorem of MacDowell and Specker on e.e.e.'s of models of Peano Arithmetic in [MS], Keisler and Morley proved that every model of ZF with countable cofinality has an e.e.e. Later [KS] dashed the hopes of a complete analogy with arithmetic by providing ad hoc examples of models of set theory with no elementary end extensions. In particular, they showed that the model $\left\langle R_{\kappa}, \varepsilon\right\rangle$, where $\kappa$ is the first strongly inaccessible cardinal, cannot be elementarily end extended. This did not settle the question whether for some strengthening $T$ of ZFC, every model of $T$ has an e.e.e. Kaufmann recently answered the question in the negative in $[\mathbf{K a}]$. His proof utilized $\omega_{1}$-like rather classless models (defined below). We later noticed that the property of being rather classless is sufficient to obstruct elementary end extensions (an idea crucial to the proof of Theorems 2.17 and 2.18 below) and that by using $\omega_{1}$-like e.e.e.'s of the so-called D.O. models (also defined below) one may altogether dispense with the heavy artillery of rather classless models in Kaufmann's theorem. Indeed, our proofs show something stronger: If $\mathfrak{A}$ is either rather classless or an $\omega_{1}$-like e.e.e. of a D.O. model, then $\mathfrak{A}$ has no rank extension satisfying ZFC. Note that although, as mentioned earlier, the model $\left\langle R_{\kappa}, \varepsilon\right\rangle$ (where $\kappa$ is the first strongly inaccessible cardinal) has no e.e.e.'s, it may very well have a rank extension satisiying ZFC, e.g., if there are two strongly inaccessible cardinals.

We now give the definitions of rather classless and D.O. models and state their existence theorems. 
Definition 1.1. A subset $X \subseteq A$ is a class of $\mathfrak{A}$ if for all $a$ in $A, X \cap a_{E}=b_{E}$ for some $b \in A . \mathfrak{U}$ is rather classless if all classes of $\mathfrak{U}$ are first-order definable (with parameters) in $\mathfrak{A}$.

The following is a specific instance of a theorem first proven by Keisler and Kunen using $\diamond_{\omega_{1}}$ in [K3]. Later Shelah [S] eliminated $\diamond_{\omega_{1}}$ by an absoluteness argument.

TheOREM 1.2 (KeISLER - KUNEN - Shelah). Every countable model of ZF has an $\omega_{1}$-like rather classless elementary end extension.

Definition 1.3. A model $\mathfrak{A}$ of set theory is a D.O. model if every ordinal of $\mathfrak{A}$ is first-order definable in $\mathfrak{A}$.

D.O. models were first introduced by Paris in [P]. He gave the following existence and uniqueness theorem. The proof given in $[\mathbf{P}]$ of Theorem 1.4 can be considerably shortened by quoting, rather than reproving, the Henkin-Orey omitting types theorem.

THEOREM 1.4 (PARIS). Every consistent extension T of ZF has a D.O. model. Moreover, if $T$ is complete, $T$ has a unique D.O. model iff $T \vdash$ "V $=$ HOD".

The main result of this section is

THEOREM 1.5. A model $\mathfrak{A} \vDash \mathrm{ZFC}$ has no rank extension satisfying ZFC if it satisfies one of the following conditions.

(a) $\mathfrak{A}$ is rather classless.

(b) $\mathfrak{A}$ is an $\omega_{1}$-like e.e.e. of a D.O. model.

Proof of (a). Assume on the contrary and suppose $\mathfrak{A}$ is rather classless and $\mathfrak{A} \subset \mathfrak{P} \vDash \mathrm{ZFC}$. Using the reflection theorem in $\mathfrak{B}$ let $\alpha \in \operatorname{Ord}(\mathfrak{B}) \backslash A$ such that $\mathfrak{P}_{\alpha} \preccurlyeq \mathfrak{B}$ and pick some $c \in B_{\alpha} \backslash A$. Using choice in $\mathfrak{P}$ we may fix a well-ordering $\triangleleft$ of $B_{\alpha}$ and in $\mathfrak{P}$ define for each $\beta<\alpha$ the model $\mathfrak{夭}_{\beta}<\mathfrak{F}_{\alpha}$ by defining $C_{\beta}$ as the collection of all elements of $R_{\alpha}$ first-order definable in $\left\langle R_{\alpha}, \varepsilon, \triangleleft\right\rangle$, using parameters from $R_{\beta} \cup\{c\}$. Now from the outside, define $\mathbb{S}=\cup_{\beta \in \operatorname{Ord}(\mathscr{2})} \mathfrak{S}_{\beta}$. At this point we have

$$
\mathfrak{U} \underset{e}{\subset} \mathfrak{C} \prec \mathfrak{P}_{\alpha} \prec \mathfrak{B} .
$$

If $\operatorname{Ord}(\mathbb{E}) \backslash A$ has a minimum element $\gamma$ then we reach a contradiction by the following trick of Kaufmann: Since $\mathfrak{C} \preccurlyeq \mathfrak{B}$, $\sqrt{ }$ is strong enough to define the full satisfaction class of $\mathfrak{A}=\mathfrak{C}_{\gamma}$, i.e., the collection $\operatorname{Sat}(\mathfrak{U})$ defined as

$$
\operatorname{Sat}(\mathfrak{U})=\left\{\langle\varphi, \vec{a}\rangle: \mathfrak{E} \vDash\left(\left\langle R_{\gamma}, \varepsilon\right\rangle \vDash \varphi[\vec{a}]\right)\right\} .
$$

But Sat $(\mathfrak{U})$ is easily seen to be a class of $\mathfrak{A}$ and hence definable in $\mathfrak{A}$, contradicting a version of Tarski's theorem on undefinability of truth.

On the other hand, if $\operatorname{Ord}(\mathfrak{E}) \backslash A$ has no minimum element we argue as follows. First consider the set $\Sigma$ defined as

$$
\Sigma=\left\{\varphi(x, \vec{a}): \vec{a} \in A \text { and } \mathfrak{B} \vDash\left(\left\langle R_{\alpha}, \varepsilon, \triangleleft\right\rangle \vDash \varphi(c, \vec{a})\right)\right\} .
$$


Here $\varphi(x, \bar{y})$ is a formula, in the sense of $\mathfrak{s}$, in the language $\{\varepsilon, \triangleleft\}$. So we may think of $\Sigma$ as a one-type, i.e., the type of $c$ in $\left\langle R_{\alpha}, \varepsilon, \triangleleft\right\rangle$ over $A$. Now the crucial idea is that $\Sigma$ is definable in $\mathfrak{A}$ by virtue of being a class of $\mathfrak{H}$. This allows us to "talk about" "단 within $\mathfrak{A}$. In particular, we may define the set $\Gamma$ in $\mathfrak{A}$ as

$$
\Gamma=\left\{t(x, \vec{a}):{ }^{\ulcorner} t(x, \vec{a}) \text { is an ordinal }{ }^{\urcorner} \in \Sigma \text { and } \forall b^{\ulcorner} t(x, \vec{a}) \neq b^{\urcorner} \in \Sigma\right\} .
$$

Here $t(x, \vec{y})$ is a (definable) term in the language $\{\varepsilon, \triangleleft\}$ in the sense of $\mathfrak{H}$, and $\ulcorner\varphi\urcorner$ codes $\varphi$. Hence $\Gamma$ corresponds to $\operatorname{Ord}(\sqrt{5}) \backslash A$.

Invoking our assumption on the absence of any minimum element in $\operatorname{Ord}(\mathfrak{S}) \backslash A$, we have

$$
\mathscr{U} \vDash(\forall t \in \Gamma)\left(\exists t^{\prime} \in \Gamma\right)\left(r^{\ulcorner} t^{\prime} \in t^{\urcorner} \in \Sigma\right) .
$$

But $\mathfrak{H}$ has a definable universal choice function since in $\mathscr{*}$ we may fix any choice function $g$ on some $R_{\theta}$, where $\theta$ is in $\operatorname{Ord}(\mathfrak{P}) \backslash A$, and then verify that $\left\{\left\langle a, a^{\prime}\right\rangle \in A^{2}\right.$ : $\left.g(a)=a^{\prime}\right\}$ is a class of $\mathfrak{A}$, and hence definable. Therefore $(*)$ implies

$$
\mathfrak{H} \vDash \exists f \forall n<\omega^{\top} f(n+1) \in f(n)^{\urcorner} \in \Sigma
$$

which, in turn, by absoluteness of $\omega$ between $\mathscr{H}$ and $\mathscr{P}$, implies

$$
\mathcal{H} \vDash \exists f \forall n<\omega\left(\left\langle R_{\alpha}, \varepsilon, \triangleleft\right\rangle \vDash f(n+1)(c) \in f(n)(c)\right),
$$

contradicting the well-foundedness of $R_{\alpha}$ in $x_{i}$.

REMARK 1.6. We remark in passing that the above proof shows that no model $\mathscr{H} \vDash \mathrm{ZFC}$ has a conservative rank extension to another model $\mathcal{H} \vDash \mathrm{ZFC}$. ( $\mathcal{H}$ is a conservative rank extension of $\mathfrak{A}$ if the intersection of any first-order definable (with parameters) subset of $B$ with $A$ is itself first-order definable with parameters.) This was also noticed by Kaufmann in "Added in Proof" of [Ka]. This is of interest since every model of $P A$, by the MacDowell-Specker theorem, has a conservative e.e.e., and in $P A$ nonconservative extensions are harder to come by than conservative ones. See e.g. R. G. Phillips [Ph].

Proof of Theorem 1.5(b). Suppose $\mathfrak{H}^{\prime}$ is D.O. and $\mathfrak{H}^{\prime} \underset{e}{\prec} \mathfrak{H}$ where $\mathscr{H}$ is $\omega_{1}$-like. Assume on the contrary that

$$
\mathfrak{H} \subset \underset{r}{ } \mathfrak{W} \vDash \mathrm{ZFC} .
$$

At this point we quote a result of Kaufmann (Theorem 3.3 of [Ka]): Any $\omega_{1}$-like model $\mathfrak{A}$ with an e.e.e. $\mathfrak{H}^{\prime}$ has an e.e.e. $\mathfrak{F}$ such that $\operatorname{Ord}(\mathfrak{F}) \backslash A$ has a minimum element. The proof given by Kaufmann proves the stronger result: If an $\omega_{1}$-like model $\mathfrak{A}$ of $\mathrm{ZFC}$ has a rank extension $\mathfrak{B} \vDash \mathrm{ZFC}$ such that $\min (\operatorname{Ord}(\mathfrak{B}) \backslash A)$ does not exist, then $\mathfrak{A}$ has an e.e.e. $\mathfrak{B}_{1}$ such that $\min \left(\operatorname{Ord}\left(\mathfrak{B}_{1}\right) \backslash A\right.$ ) exists ( $\mathfrak{H}$ will also have an e.e.e. $\mathfrak{P}_{2}$ such that $\min \left(\operatorname{Ord}\left(\mathfrak{B}_{2}\right) \backslash A\right)$ does not exist). Hence without loss of generality, for some $\gamma \in \operatorname{Ord}(\mathfrak{B}), \mathfrak{A}=\mathfrak{B}_{\gamma}$. But then arguing in $\mathfrak{B}$, we note that every member of $\omega_{1}$ is definable in $\left\langle R_{\gamma}, \varepsilon\right\rangle$, implying that $\omega_{1}$ is countable!

REMARK 1.7. One cannot strengthen Theorem 1.5 by weakening "rank extension" to "end extension". This can be easily seen by a forcing argument. Since most expositions of forcing concentrate on generic extensions of standard models, we briefly elaborate. Given any model $\mathfrak{A}$ of set theory and any Boolean algebra $\mathbf{B}$, 
complete in the sense of $\mathfrak{A}$, we denote the B-valued model, $V^{\mathbf{B}}$ of $\mathfrak{A}$, by $\mathfrak{A}^{\mathbf{B}}$ (see [J, $\S 18])$. If $G$ is any ultrafilter over $\mathbf{B}$ we may then form the reduced two-valued model $\mathfrak{A}^{\mathbf{B}} / G$ in the usual way. This model, in general, is not an end extension of $\mathfrak{A}$, but if $G$ is B-generic over $\mathfrak{A}$, then, in view of the equation

$$
\|x \in y\|=\sum_{t \in \operatorname{dom}(y)}(\|x=t\| \cdot y(t)),
$$

$\mathfrak{A}^{\mathbf{B}} / G$ will be an end extension of $\mathfrak{A}$. Also, given any such $\mathbf{B}$ in an $\omega_{1}$-like model $\mathfrak{A}$ we may always find an ultrafilter which is $\mathbf{B}$ generic over $\mathfrak{A}$. Since, by Theorem 1.2, $\omega_{1}$-like rather classless models exist and, by $\omega_{1}$ applications of the Keisler-Morley theorem, every D.O. model has an $\omega_{1}$-like e.e.e., we conclude that "rank extension" cannot be replaced by "end extension" in Theorem 1.5.

2. Large cardinal characterizations. In this section we provide characterizations of certain large cardinal properties in terms of elementary extensions. We start with some preliminaries. Note that in this section "definable" always means first order with parameters.

Definition 2.1. A ranked tree is a structure $\left\langle T,<_{T}, O,<_{0}, r\right\rangle$ such that

(i) $\left\langle T,<_{T}\right\rangle$ is a tree, i.e. a partial order whose initial segments (determined by nodes) are linearly ordered;

(ii) $\left\langle O,<_{0}\right\rangle$ is a linear order with no last element; and

(iii) $r$ is an order-preserving map from $\left\langle T,<_{T}\right\rangle$ onto $\left\langle O,<_{0}\right\rangle$ i.e. $s<_{T} t \rightarrow r(s)$ $<{ }_{0} r(t)$.

A subset $B \subseteq T$ is a branch if $r$ maps $B$ onto $O$. Given a model $\mathfrak{A}$ and some ranked tree $T=\left\langle T,<_{T}, \ldots\right\rangle$ definable in $\mathfrak{A}$ we say that $T$ is rather branchless in $\mathfrak{A}$ if every branch of $T$ is definable in $\mathfrak{A}$.

We heavily use the following result which, in the present form, is due to Shelah, [S, Theorem 12] who eliminated $\diamond_{\omega_{1}}$ and generalized Theorem B of [K3].

THEOREM 2.2 (SHELAH). Every countable model in a countable language has an elementary extension of power $\aleph_{1}$ in which every definable tree is rather branchless.

As our first application we have

THEOREM 2.3. Let $\kappa$ be a distinguished constant of a theory $T \supseteq$ ZFC. The following are equivalent.

(i) $T \vdash$ " $\kappa$ is weakly compact".

(ii) $T \vdash$ " $\kappa$ is strongly inaccessible" and given any $\mathfrak{A} \vDash T$ and any $X \in \mathfrak{A}_{\kappa+1}$ the model $\left\langle\mathfrak{A}_{\kappa}, X\right\rangle$ has an e.e.e.

ProOF. (i) $\Rightarrow$ (ii). Recall the Keisler characterization of weak compactness: $\kappa$ is weakly compact iff $\kappa$ is strongly inaccessible and for every $X \subseteq R_{\kappa}$ the model $\left\langle R_{\kappa}, X\right\rangle$ has an e.e.e. Hence this is the easy direction.

(ii) $\Rightarrow$ (i). Assume on the contrary. Thus $T \cup\{$ “ $\kappa$ is not weakly compact" $\}$ is consistent and has a countable model $\mathfrak{A}$. Let $\mathfrak{B}=\langle B, F, \ldots\rangle$ be any elementary extension of $\mathfrak{A}$ in which the binary (ranked) tree of height $\kappa$ is rather branchless. Note that this means that whenever $X \subseteq \kappa_{F}$ and $X \cap \gamma \in B$ for every $\gamma \in \kappa_{F}$ then 
$X \in B$. On the other hand, by the tree characterization of weakly compacts we know that for some $\tau \in \mathbb{R}$

$$
\mathfrak{H} \vDash \text { “ } \tau \text { is a } \kappa \text {-Aronszajn (ranked) tree". }
$$

Now $\tau$ can be obviously coded in $R_{\kappa}$ and we shall confuse $\tau$ with its code, so applying (ii) the model $\left\langle\mathfrak{P}_{\kappa}, \tau\right\rangle$ has an e.e.e. $\left\langle\mathcal{S}, \tau^{\prime}\right\rangle$. Note that $\tau^{\prime}$ properly end extends $\tau$, so if we pick some $c \in \tau^{\prime} \backslash \tau$ then $\left\{b \in \tau^{\prime}: b<{ }_{\tau^{\prime}} c\right\}$ is a branch of $\tau$, hence belonging to $\mathfrak{R}$, contradicting $(*)$.

THEOREM 2.4. Let $\kappa$ be a distinguished constant of a theory $T \supseteq \mathrm{ZFC}$ where $T \vdash " R_{\kappa} \vDash Z F C "$. The following are equivalent.

(i) $\forall n<\omega T \vdash " R_{\kappa}$ has a $\Sigma_{n}$-e.e.e."

(ii) $\forall n<\omega$ and for all $\mathfrak{A} \vDash T$ the model $\mathfrak{H}_{\kappa}$ has a $\Sigma_{n}$-e.e.e.

Proof. We prove the nontrivial direction. Given an arbitrary $n<\omega$ and any countable model $\mathfrak{A}_{0}$ of $T$ we first let $\mathfrak{A} \succ \mathfrak{H}_{0}$ be a model in which the following ranked tree $\tau$ is rather branchless. The nodes of $\tau$ are ordered pairs $(a, \alpha)$ where $\alpha<\kappa$ and $\mathfrak{A} \vDash " a \subseteq R_{\alpha}$ ". The ordering between nodes is defined in $\mathfrak{A}$ by

$$
(a, \alpha)<(b, \beta) \text { iff } \alpha<\beta \text { and } b \cap R_{\alpha}=a \text {. }
$$

The $\tau$-rank of $(a, \alpha)$ is naturally $\alpha$. It is easy to show that if $T$ is rather branchless in $\mathfrak{U}$ then every class (see Definition 1.1 ) of $\mathfrak{A}$, belongs to $\mathfrak{A}$.

Using (ii), $\mathfrak{A}_{\kappa}$ has some $\Sigma_{n+1}$-e.e.e. $\mathfrak{P}$, hence by the (relativized) reflection theorem we may choose some $\gamma \in \operatorname{Ord}(\mathfrak{B}) \backslash A_{\kappa}$ such that $\mathfrak{B}_{\gamma} \prec \mathfrak{B}$ and then pick some $c \in B_{\gamma} \backslash A_{\kappa}$ and some $\triangleleft$ such that $\mathfrak{P} \vDash$ " $\triangleleft$ well-orders $R_{\gamma}$ ". Now consider

$$
\Gamma=\left\{\varphi(x, \vec{a}): \vec{a} \in A_{\kappa} \text { and } \mathfrak{P} \vDash "\left\langle R_{\gamma}, \varepsilon, \triangleleft\right\rangle \vDash \varphi(c, \vec{a}) "\right\} .
$$

Here $\varphi$ is a formula in the sense of $\mathfrak{B}$ in the language $\{\varepsilon, \triangleleft\}$. $\Gamma$ is easily seen to be a class of $\mathfrak{A}_{\kappa}$. Hence by our choice of $\mathfrak{A}, \Gamma$ belongs to $\mathfrak{A}$. The reader may verify that $\mathscr{H} \vDash$ " $R_{\kappa}$ has a $\Sigma_{n}$-e.e.e." Therefore $\mathfrak{H}_{0}$ says the same thing. In view of the completeness theorem the proof is complete.

REMARK 2.5. One may guess that if for every model $\mathfrak{A}$ of a certain theory $T \supseteq$ ZFC the model $\mathfrak{A}_{\kappa}$ has an e.e.e. then $T \vdash$ " $R_{\kappa}$ has an e.e.e." This, however, need not be true since the theory $T_{0}=\mathrm{ZFC}+\left\{“ R_{\kappa}{ }_{n} V\right.$ ": $\left.n<\omega\right\}+\left\{\right.$ " $R_{\kappa}$ has no e.e.e." $\}$ $+\{$ " $\kappa$ is inaccessible" $\}$ is consistent (relative to a weakly compact). This follows from Theorem 4.4 of [Ka]. Note that $T_{0}$ has no well-founded models since, by an observation of Kunen, if $\mathfrak{M} \prec \mathfrak{M} \vDash Z$ ZFC, $\mathfrak{Y}$ is well founded and $\mathfrak{M}=\mathfrak{M}_{\gamma}$ then $\mathfrak{H} \vDash$ " $R_{\gamma}$ has an e.e.e." For a sketch see the proof of Theorem 2.5 of [Ka]. Indeed, in contrast with other results of this section we have

THEOREM 2.6 (KUnen). Assume there exists a weakly compact cardinal. There is no consistent theory $\Phi$ such that for all consistent $T \supseteq \mathrm{ZFC}, T \vdash \Phi$ iff for every model $\mathfrak{A}$ of $T, \mathfrak{A}_{\kappa}$ has an e.e.e.

Proof. Assume to the contrary and let $\Phi$ be such a theory. We claim that ZFC $+\Gamma \vDash \Phi$ where $\Gamma=\left\{\right.$ “ $R_{\kappa}$ has a $\Sigma_{n}$-e.e.e.": $\left.n<\omega\right\}$. If not, then for some $\varphi \in \Phi$ the theory $T_{0}=\mathrm{ZFC}+\Gamma+\neg \varphi$ is consistent. Since $T_{0}$ is a recursive theory there is 
some sentence $\neg \operatorname{Con}\left(T_{0}\right)$ which expresses the inconsistency of $T_{0}$, hence $T_{1}=T_{0}+$ $\neg \operatorname{Con}\left(T_{0}\right)$ is a consistent theory with no $\omega$-model. Therefore if $\mathfrak{M} \vDash T_{1}$ then by overspill there is an infinite integer $H$ of $m$ such that

$$
\mathfrak{M} \vDash \text { “ } R_{\kappa} \text { has a } \Sigma_{H^{-}} \text {e.e.e." }
$$

In particular, for every model $\mathfrak{M}$ of $T_{1}$ the model $\mathfrak{M}_{\kappa}$ will have an e.e.e. Hence $T_{1} \vdash \Phi$, which contradicts the fact that $\neg \varphi \in T_{1}$. At this point we know that every model of ZFC and $\Gamma$ is also a model of $\Phi$, so, by exhibiting a model $\mathfrak{A}$ of $\Gamma$ such that $\mathfrak{A}_{\kappa}$ has no e.e.e., we would reach our final contradiction. Let $\kappa$ be the first strongly inaccessible cardinal witnessing $\Gamma$ in $V$ (which exists since we are assuming there exists a weakly compact cardinal). By Theorem 1.2 of $[\mathrm{Ka}],\left\langle R_{\kappa}, \varepsilon\right\rangle$ has no e.e.e.'s which, in turn, implies that $\kappa$ is smaller than the first weakly compact cardinal. In particular, $\kappa<\gamma$ for some strongly inaccessible $\gamma$, hence if $\mathfrak{A}$ is chosen to be $\left\langle R_{\gamma}, \varepsilon, \kappa\right\rangle$, our proof will be complete.

We now prove some results concerning measurable cardinals, but first

Definition 2.7. $\mathfrak{B}$ is a $\kappa$-elementary end extension of $\mathfrak{A}$ (hereafter abbreviated as $\kappa$-e.e.e.) if $\kappa \in A$ and $\mathfrak{B}$ enlarges $\kappa$ without enlarging any members of $\kappa$. Note that if $\mathfrak{U}$ has a $\kappa$-e.e.e. then $\mathfrak{A} \vDash$ “ $\kappa$ is regular". The oldest theorem concerning $\kappa$-e.e.e.'s is the following result implicit in [Sc].

THEOREM 2.8. Let $\mathfrak{A} \vDash$ ' $\kappa$ is a measurable cardinal ( $\omega$ included)'. Then $\mathfrak{A}$ has a к-e.e.e.

Remark 2.9. When $\mathfrak{A} \vDash$ " $\kappa>\omega$ " the usual (Scott ultrapower) proof of Theorem 2.8 yields a $\kappa$-e.e.e., $\mathfrak{B}$, of $\mathfrak{A}$ in which $\kappa$ has a least new member. In view of Theorem 2.12 below it is worth mentioning that by iterating the ultrapower $\omega$ times "from outside" and within $\mathfrak{A}$, of course, we get a $\kappa$-e.e.e. of $\mathfrak{A}$ in which $\kappa$ has no least new member.

$\kappa$-elementary end extensions are closely linked to ultrafilters; to make this connection exact we need

Definition 2.10. Let $\mathfrak{A} \vDash Z F C$ and $\kappa$ be a cardinal of $\mathfrak{A}$. $\mathscr{Q}$ is said to be a $\kappa$-complete ultrafilter over $\mathfrak{U}$ if

(1) $\mathcal{Q}$ is a nonprincipal ultrafilter over the Boolean algebra of $\mathfrak{A}$-subsets of $\kappa$;

(2) $\mathscr{Q}$ is $\kappa$-complete over $\mathfrak{A}$ : whenever $\left\langle X_{\alpha}: \alpha<\lambda<\kappa\right\rangle \in A$ and $X_{\alpha} \in \mathcal{U}$ then $\cap_{\alpha<\lambda} X_{\alpha} \in \mathcal{Q}$. Note that this is equivalent to: For every partition $p$ of $\kappa$ in $\mathfrak{A}$ of size less than $\kappa, p \cap \mathcal{U}$ is nonempty.

THEOREM 2.11 [K1]. The following are equivalent.

(i) $\mathfrak{U}$ has a k-e.e.e.

(ii) $\mathfrak{A}$ has $a \Pi_{2}$-к-e.e.e.

(iii) There exists a к-complete over $\mathfrak{A}$ ultrafilter.

Theorem 2.11 allows us to prove

THEOREM 2.12. Let $\mathfrak{A}=\langle A, E, \ldots\rangle \vDash \mathrm{ZFC}$ and $\kappa$ be a regular cardinal of $\mathfrak{A}$ such that $\left(2^{\kappa}\right)_{E}$ is countable. Then $\mathfrak{A}$ has a $\kappa$-e.e.e., $\mathfrak{B}$. Moreover, if $\kappa$ is uncountable in $\mathfrak{A}$ then $\mathfrak{B}$ may be required to either have a least new member of $\kappa$ or have no least new member of $\kappa$. 
Theorem 2.12 generalizes a similar result of [KM and H1] where $\mathfrak{H}$ itself was assumed to be countable (cf. also Chapter 33 of [K2]).

Proof (SKeTCH). We outline an efficient generic ultrapower formulation. Consider the following partial orders in $\mathfrak{A}$ :

$$
\begin{aligned}
& \mathbf{P}=\{X \subseteq \kappa: X \text { is stationary }\} ; \\
& \mathbf{Q}=\{X \subseteq \kappa:|X|=\kappa\},
\end{aligned}
$$

where the ordering in both cases is set inclusion. Consider the sets in $\mathfrak{H}$ of the form $D_{f}=\{X \subseteq \kappa: f \uparrow X$ is constant $\}$ where $f$ is a regressive function on $\kappa$ in $\mathscr{A}$. Each $D_{f}$ is a dense subset of $\mathbf{P}$ by the Pressing down lemma. Hence, by our assumption of countability of $2^{\kappa}$ of $\mathfrak{A}$ there is an ultrafilter $G_{1}$ which meets all the $D_{f}$ 's. It is easy to check that the ultrapower $\mathfrak{P}_{1}=\mathfrak{A}^{\kappa} / G_{1}$ is a $\kappa$-e.e.e. with a least new member of $\kappa$. On the other hand, we may look at sets in $\mathfrak{A}$ of the form

$$
E_{f}=\{X \subseteq \kappa: \exists g: \kappa \rightarrow \kappa, g<f \text { on } X \text { and } g \text { is one-to-one on } X\}
$$

where

$$
\mathfrak{U} \vDash " f: \kappa \rightarrow \kappa \quad \text { and } \quad \forall \alpha<\kappa\left|f^{-1}(\{\beta \in \kappa: \beta>\alpha\})\right|=\kappa " .
$$

$E_{f}$ is dense in $\mathbf{Q}$ by a simple simultaneous induction argument. Also by the regularity of $\kappa$ if $h: \kappa \rightarrow \kappa$ has bounded image then sets of the form $K_{h}=\{X \subseteq \kappa: h \uparrow X$ is constant $\}$ are also dense in $\mathbf{Q}$. The reader may verify that if $G_{2}$ is any ultrafilter meeting all $E_{f}$ 's and $K_{h}$ 's, then $\mathfrak{B}_{2}=\mathfrak{A}^{\kappa} / G_{2}$ is a $\kappa$-e.e.e. of $\mathfrak{A}$ with arbitrary small new members of $\kappa$. It is worth mentioning that both $\mathfrak{H}_{1}$ and $\mathfrak{X}_{2}$ are minimal $\kappa$-e.e.e.'s of $\mathfrak{A}$ in the sense that if for some model $\mathfrak{S}$ we have $\mathfrak{U} \prec \mathfrak{S} \prec \mathfrak{i}$ then $\mathfrak{S}=\mathfrak{H}$ or $\mathfrak{C}=\mathfrak{B}$ where $\mathfrak{P}=\mathfrak{B}_{1}$ or $\mathfrak{B}_{2}$.

Remark 2.13. If $\mathfrak{U} \vDash$ "There exists an $\omega_{1}$-Kurepa tree" and $\omega$ of $\mathfrak{H}$ is countable but $\omega_{2}$ of $\mathfrak{H}$ is uncountable, then $\mathfrak{A}$ has no $\omega_{1}^{\mathfrak{H}}$-e.e.e.'s. Therefore, the condition of countability of $2^{\kappa}$ of $\mathfrak{A}$ in Theorem 2.12 cannot be weakened to countability of $\kappa$ itself.

THеонем 2.14. Let $T \supseteq \mathrm{ZFC}$ and $\kappa$ be a distinguished constant of $T$. Then, assuming either (a) $T \vdash 2^{\kappa}=\kappa^{+}$or (b) $\diamond_{\omega_{1}}$ (in the real world), the following are equivalent.

(i) $T \vdash$ " $\kappa$ is a measurable cardinal".

(ii) Every model of $T$ has a -e.e.e.

Proof. The proof using (a) is due to the author, and the one using (b) is due to $\mathrm{M}$. Rubin. Rubin's proof is more powerful than ours and it led us to the proof of Theorems 2.17 and 2.18 below. We outline the proof of the nontrivial direction using (a). Assume on the contrary and let $\mathfrak{A}$ be a countable model of $T+$ " $\kappa$ is not measurable". Using Theorem 2.2 let $\mathfrak{B} \succ \mathfrak{A}$ in which the binary (ranked) trees of height $\kappa$ and $\kappa^{+}$are rather branchless. It is not hard to see that (ii) implies that $T \vdash$ ' $\kappa$ is strongly inaccessible'. Now if $\mathfrak{B}$ has a $\kappa$-e.e.e., 5 , then we may pick a new member $c$ of $\kappa$ and consider the set

$$
\text { थl }=\{X \in B:(\mathfrak{S} \vDash(c \in X \subseteq \kappa)\} .
$$


To show that $\mathscr{Q} \in B$, it suffices (since $2^{\kappa}=\kappa^{+}$) to show that given any sequence $\left\langle X_{\alpha}: \alpha<\kappa\right\rangle$ of subsets of $\kappa$ in $\mathfrak{B}$ the set $M=\left\{\alpha<\kappa: X_{\alpha} \in \mathscr{U}\right\}$ is in $\mathfrak{B}$. But $M$ is in $\mathfrak{B}$ essentially because of the fact that $\kappa$ is strongly inaccessible and the binary tree of height $\kappa$ is rather branchless in $\mathfrak{B}$. $\mathscr{Q}$ is obviously a $\kappa$-complete ultrafilter over $\mathfrak{P}$ and if $\mathscr{Q} \in B$ then $\kappa$ is measurable in $\mathfrak{B}$. This is a contradiction.

The reader should convince himself that the same method of proof shows that condition (a) can be relaxed to

$$
\text { For some } n<\omega, T \vdash “ 2^{\kappa} \leqslant \overbrace{}^{n \text {-times }},
$$

This was pointed out to us by Boban Velickovič.

To present the proof using (b), we first need to quote a theorem of Rubin (see [RS, Corollary 2.4 and Theorem 2.5]).

THEOREM $2.15\left(\diamond_{\omega_{1}}\right)$ (RUBIN). Let $\mathfrak{A}$ be a countable model in a countable language. There exists $\mathfrak{P}>\mathfrak{A}$ such that:

(I) Every directed set definable in $\mathfrak{B}$ with no last element has a cofinal chain of length $\omega_{1}$.

(II) If $P$ is a definable partial order of $\mathfrak{P}$ and $X \subseteq P$ is maximal compatible and contains a cofinal chain (in $X$ ) of length $\omega_{1}$ then $X$ is definable ( $p$ and $q$ are compatible if $\exists r>p, q$ ).

REMARK 2.16. In Theorem 2.15 if $\mathfrak{A}$ is a model of $\mathrm{ZF}$ then (I) and (II) together imply that every tree in $\mathfrak{B}$ is rather branchless. In particular, $\mathfrak{B}$ would be rather classless.

We are now ready to give a proof of Theorem 2.14 using (b). Assume on the contrary and let $\mathfrak{A}$ be a countable model of ' $\kappa$ is not measurable' $+T$. Let $\mathfrak{P}>\mathfrak{A}$ be as in Theorem 2.15. By Theorem 2.11, and assumng (ii), there exists an ultrafilter $\mathcal{U}$ which is $\kappa$-complete over $\mathfrak{B}$. To show that $\mathscr{Q} \in B$ we first use (I) to choose a cofinal sequence $\left\langle d_{\alpha}: \alpha<\omega_{1}\right\rangle$ in the directed set of all finite partitions of $\kappa$ in the sense of $\mathfrak{U}$ (directed by the refinement relation). Since $\mathcal{U}$ is $\kappa$-complete over $\mathfrak{U}$ there exists a unique $p_{\alpha}$ in $\mathscr{Q} \cap d_{\alpha}$. It is easy to see that $p_{\alpha} \supseteq p_{\beta}$ whenever $\alpha<\beta$ and that the sequence $\left\langle p_{\alpha}: \alpha<\omega_{1}\right\rangle$ is cofinal in $\mathcal{U}$ (where $\mathcal{U}$ is partially ordered by reverse inclusion). Thus, in view of (II) and the fact that $\mathcal{Q}$ is a maximal compatible subset of the partially ordered set of all nonempty subsets of $\kappa$ (ordered by reverse inclusion), $\mathcal{Q}$ must belong to $B$. But then $\kappa$ would be measurable in $\mathfrak{B}$. This is a contradiction.

THEOREM $2.17\left(\diamond_{\omega_{1}}\right)$. Let $T \supseteq \mathrm{ZFC}$. The following are equivalent.

(i) $T \vdash$ "There exists an uncountable measurable cardinal".

(ii) Every model $\mathfrak{A}$ of $T$ has an elementary extension which fixes $\omega^{\mathscr{N}}$.

Proof. We prove the nontrivial direction. As usual we assume on the contrary that $T+$ 'There are no uncountable measurable cardinals' is consistent and we let $\mathfrak{A}$ be a countable model of this theory. Now let $\mathfrak{P}$ be an elementary extension of $\mathfrak{A}$ as in Theorem 2.15. Using (ii), if some $\mathfrak{E} \succ \mathfrak{B}$ and $\mathfrak{E}$ fixes $\omega^{\mathfrak{B}}$, then, by Remark 2.16 
and Theorem 1.5(a), $\sqrt{5}$ must enlarge some $\lambda \in B$. So fix some new member $c$ of $\lambda$ and define the ultrafilter $\mathscr{U}$ as $\{X \in B$ : $\mathfrak{F} \vDash(c \in X \subseteq \lambda)\}$. But $\mathscr{Q}$ must be in $\mathfrak{P}$ by an argument similar to the proof of Theorem 2.14 using (b). Hence $\mathfrak{P} \vDash$ "Q $Q$ is an $\omega_{1}$-complete ultrafilter", therefore by a classical result of Ulam

$$
\mathfrak{P} \vDash \exists \theta>\omega(\theta \text { is measurable and } \mathscr{Q} \text { is } \theta \text {-complete }) \text {. }
$$

This is a contradiction.

With an argument similar to the previous proof we get the following result; the proof is omitted.

THEOREM $2.18\left(\diamond_{\omega_{1}}\right)$. Let $T \supseteq \mathrm{ZFC}$. The following are equivalent.

(i) $T \vdash$ "There are arbitrarily large measurable cardinals".

(ii) For every model $\mathfrak{A} \vDash T$ there are arbitrarily large $\kappa$ 's in $\operatorname{Ord}(\mathfrak{U})$ such that $\mathfrak{A}$ has a к-e.e.e.

(iii) For every model $\mathfrak{A} \vDash T$ and every $c \in A$ there exists an elementary extension which fixes $c$.

Lastly, we present two results concerning the problem of initial elementary submodels.

THEOREM 2.19. Any extension of ZF has a model with no initial elementary submodels.

Proof. Use a D.O. model.

THEOREM 2.20. Suppose $T \supseteq \mathrm{ZF}$ and for some formula $\mathfrak{M}(x), T$ proves “Mi is transitive" and $T \vdash \varphi^{\mathfrak{M}}$ for each axiom $\varphi$ of $\mathrm{ZF}$. Assuming $T$ is complete the following are equivalent.

(i) For every $\mathfrak{A} \vDash T, \mathfrak{M}^{\mathfrak{A}}$ has an initial elementary submodel.

(ii) There are formulas $\psi(x), \varphi_{n}(x), n<\omega$, such that $T$ proves:

(a) $\exists ! x\left(\varphi_{n}(x)\right.$ and $x$ is an ordinal $), n<\omega$;

(b) $\forall x \varphi_{n}(x) \rightarrow$ “M $\mathfrak{M}_{x} \prec \mathfrak{M} ", n<\omega$;

(c) $\forall x \forall y\left(\varphi_{n}(x) \wedge \varphi_{m}(y) \rightarrow x \in y\right), n<m<\omega$;

(d) $\exists y \psi(y)$ and $\forall x \forall y\left(\varphi_{n}(x) \wedge \psi(y) \rightarrow x \in y\right), n<\omega$.

PROOF. Use D.O. models and the reflection theorem in $\mathfrak{M}$ for the nontrivial direction.

REMARK 2.21. Note that if $T_{0}=\mathrm{ZF}+$ " $O^{\#}$ exists", and $\mathfrak{M}(x)=$ " $x$ is constructible", then $T_{0}$ satisfies (i) above. In light of $\S 1$ it is curious that by stretching Silver indiscernibles the constructible universe of every model of $T_{0}$ has an e.e.e.

ACKNOWLEDGEMENTS. I wish to thank M. Rubin for his never ending interest in this work, and discussions concerning his and Shelah's results. I am also indebted to Ken Kunen for his patient ear and useful comments.

\section{REFERENCES}

[E] A. Enayat, On certain elementary extensions of models of set theory, Abstracts Amer. Math. Soc. 4 (1983), 310.

[H1] J. E. Hutchinson, Elementary extensions of countable models of set theory, J. Symbolic Logic 41 (1976), 139-145.

[H2] , Model theory via set theory, Israel J. Math. 24 (1976), 286-303. 
[J] T. Jech, Set theory, Academic Press, New York, 1978.

[Ka] M. Kaufmann, Blunt and topless end extensions of models of set theory, J. Symbolic Logic 48 (1983), 1053-1073.

[K1] H. J. Keisler, Extending models of set theory, J. Symbolic Logic 30 (1965), 269 (Abstract).

[K2] _ Model theory for infinitary logic, North-Holland, Amsterdam, 1971.

[K3] _ Models with tree structures (Proc. Tarski Sympos., 1971), Proc. Sympos. Pure Math., vol. 25, Amer. Math. Soc., Providence, R.I., 1974, pp. 331-348.

[KM] H. J. Keisler and M. Morley, Elementary extensions of models of set theory, Israel J. Math. 5 (1968), 49-65.

[KS] H. J. Keisler and J. Silver, End extensions of models of set theory, Axiomatic Set Theory. Part I, Proc. Sympos. Pure Math., vol. 13, Amer. Math. Soc., Providence, R.I., 1970, pp. 177-187.

[MS] R. MacDowell and E. Specker, Modelle der Arithmetik, Infinitistic Methods (Warsaw, 1959), Proc. Sympos. Found. Math., Pergamon Press, New York, and Oxford, 1961, pp. 257-263.

[P] J. B. Paris, Minimal models of ZF, Proceedings of the Bertrand Russell Memorial Logic Conference (Denmark, 1971), Leeds, 1973, pp. 327-331.

[Ph] R. G. Phillips, Omitting types in arithmetic and conservative extensions, Victoria Symposium on Nonstandard Analysis (A. Hurd and P. Loeb, eds.), Lecture Notes in Math., Vol. 369, Springer-Verlag, Berlin and New York, 1974, pp. 195-202.

[RS] M. Rubin and S. Shelah, On the elementary equivalence of automorphism groups of Boolean algebras, downward Skolem-Lowenhiem theorems and compactness of related quantifiers, J. Symbolic Logic 45 (1980), 265-283.

[Sc] D. Scott, Measurable cardinals and constructible sets, Bull. Acad. Polon. Sci. Sér. Sci. Phys. Astron. 9(1961), 521-524.

[S] S. Shelah, Models with second order properties. II. Trees with no undefined branches, Ann. Math. Logic 14 (1978), 73-87.

Department of Mathematics, University of Wisconsin, Madison, Wisconsin 53706 